\title{
Regional Distribution of Acetylcholinesterase in the Right Atria of Humans and Dogs
}

\author{
SACHCHIDA N. SINHA, MICHAEL R. YELICH, STEVEN KERESZTES-NAGY, AND ALLEN \\ FRANKFATER \\ Departments of Pediatrics and Biochemistry, Loyola University Stritch School of Medicine, Maywood, Illinois, and \\ Department of Pediatrics, Peoria School of Medicine, University of Illinois, Peoria, Illinois, USA
}

\begin{abstract}
Summary
The regional distribution of acetylcholinesterase in the right atrium was determined by quantitative chemical measurements on hearts obtained from 14 infant and 9 adult humans at autopsy, and 9 adult dogs after termination of acute animal experiments. The atrium and interatrial septum were dissected, and the appendage was cut along its fold from the ventricular border to the superior vena cava. The atrium was cut into 20 consecutive sections. Homogenates $(10 \% \mathrm{w} / \mathrm{v})$ were prepared, centrifuged, and the supernatants were used for the enzyme assay by the method of Ellman. The acetylcholinesterase concentration $[\mathrm{AChE}]$ was mapped by section, or the sections were grouped into areas and mapped. The results show that: 1$)$ in the dog, $[\mathrm{AChE}]$ is significantly higher in the nodal regions as compared to the appendicular areas, which contain the lowest $|A C h E| ; 2)$ in the human, the [AChE] distribution pattern is qualitatively similar between the adult and infant, and in contrast to the dog, the appendicular areas contain the highest $[\mathrm{AChE}] ; 3)$ for all areas studied, human infant [AChE] levels are significantly higher than human adult levels for corresponding areas. It is concluded that there is a distinct species difference between the regional distribution of the $[\mathrm{AChE}]$ in human and canine right atrium. Also, within humans, there is an age-related difference in the quantitative $[\mathrm{AChE}]$ levels. These species and age-related differences may reflect a varying pattern of distribution of the vagus nerve between the two species studied.
\end{abstract}

\section{Speculation}

This study presents quantitative data about the regional distribution of $\mathrm{AChE}$ concentration within the right atria of adult dog, and adult and infant human hearts. Significant species and agerelated differences were observed. Because a close correlation is known to exist between the concentration of acetylcholine, acetylcholinesterase, and choline acetylase in various regions of the peripheral and central nervous system, it is speculated that the differences in the distribution of acetylcholinesterase within the right atrium reported here reflect a difference in the pattern of cholinergic innervation between species.

AChE appears in the myocardium early during fetal development and its appearance is known to precede the functional innervation of the heart. This enzyme is responsible for hydrolyzing and inactivating acetylcholine, the transmitter substance released from parasympathetic nerve terminals. The ontogenesis of $\mathrm{AChE}$ has been well reviewed and it has been shown by histochemical studies that high enzyme activity is present in the human heart at birth (10). Recently, postnatal changes in enzyme levels per se have been investigated in the human and dog heart (11). Significant species and age-related differences in cholinesterase distribution within the heart were reported, but little is known about the relative concentration of this enzyme in selected areas of the atrium as determined by quantitative chemical measure- ment. The present work investigates, in greater regional detail, the distribution of $\mathrm{AChE}$ within the right atrium of human adults and infants, and dogs.

\section{MATERIALS AND METHODS}

Human heart tissues were obtained from autopsied material at the Cook County Coroner's Morgue. Nine adults ranging in age from $15-78 \mathrm{yr}$, and 14 infants ranging in age from 2 days to 14 months were studied. Fresh canine heart tissues were obtained from nine adult animals which had been utilized for acute openchest physiological studies. Human specimens were temporarily stored in $0.9 \%$ saline while being transported to the laboratory for immediate analysis. Animal specimens were analyzed immediately after death. The time of analysis was not critical, as AChE concentration levels remained stable in saline-stored tissues left at room temperature for up to $24 \mathrm{hr}$.

From each heart, the right atrium and interatrial septum were dissected and were meticulously sectioned and designated into 20 small pieces in a careful and consistent manner. In preparing an atrium for sectioning, it was first freed of all visible fat, and then the appendage was cut along its fold from the ventricular border to the superior vena cava. The entire chamber was arranged on and pinned to a wax slab (Fig. 1). Landmarks such as the fossa ovalis, coronary sinus, and crista terminalis aided in the consistent separation of the chamber into 20 sections as schematically represented in Figure $2 \mathrm{~A}$. The $\mathrm{AChE}$ enzyme assay was carried out on each section by the spectrophotometric method of Ellman et al. (7).

Ten percent $(w / v)$ homogenates of each tissue section were prepared with a ground-glass tissue homogenizer in phosphate buffer containing $1 \%$ Triton X-100. Homogenates were centrifuged for $10 \mathrm{~min}$ at $15,000 \mathrm{Xg}$ and the supernatants were used in the enzyme assay system. $100 \mu \mathrm{l}$ of supernatant was mixed with $3.0 \mathrm{ml}$ of $0.423 \mathrm{mM}$ DTNB (5,5'-Dithiobis-(2-Nitrobenzoate) in phosphate buffer. In addition, $25 \mu \mathrm{l}$ of iso-OMPA (tetraisopropylphosphoramide, $0.4 \mathrm{mM}$ in phosphate buffer) was added to permit differentiation of acetylcholinesterase from butylcholinesterase $(1,4,11)$. The mixture was incubated at $37^{\circ} \mathrm{C}$ for $30 \mathrm{~min}$, after which time the Ellman reaction was initiated with the addition of $25 \mu \mathrm{l}$ of acetylthiocholine iodide $(78 \mathrm{mM})$. The change in absorbance $(\Delta \mathrm{A})$ at $412 \mathrm{~nm}$ was followed manually with a Gilford 2400 spectrophotometer. Readings were taken at 2-min intervals for $6 \mathrm{~min}$, the results were averaged and corrected for the slow spontaneous decomposition of the substrate. The concentration of $\mathrm{AChE}$, [AChE], was expressed as $\Delta \mathrm{A} / \mathrm{min} \times 10^{3}$, which is proportional to international units of $\mathrm{AChE}$ (16). As a constant weight percent of homogenate was used throughout, the concentrations of acetylcholinesterase expressed in this paper are directly proportional to tissue concentrations. A more detailed account of the AChE assay has been described previously (11).

In order to simplify and facilitate the statistical analysis of the data and the description thereof, atrial sections were grouped into 


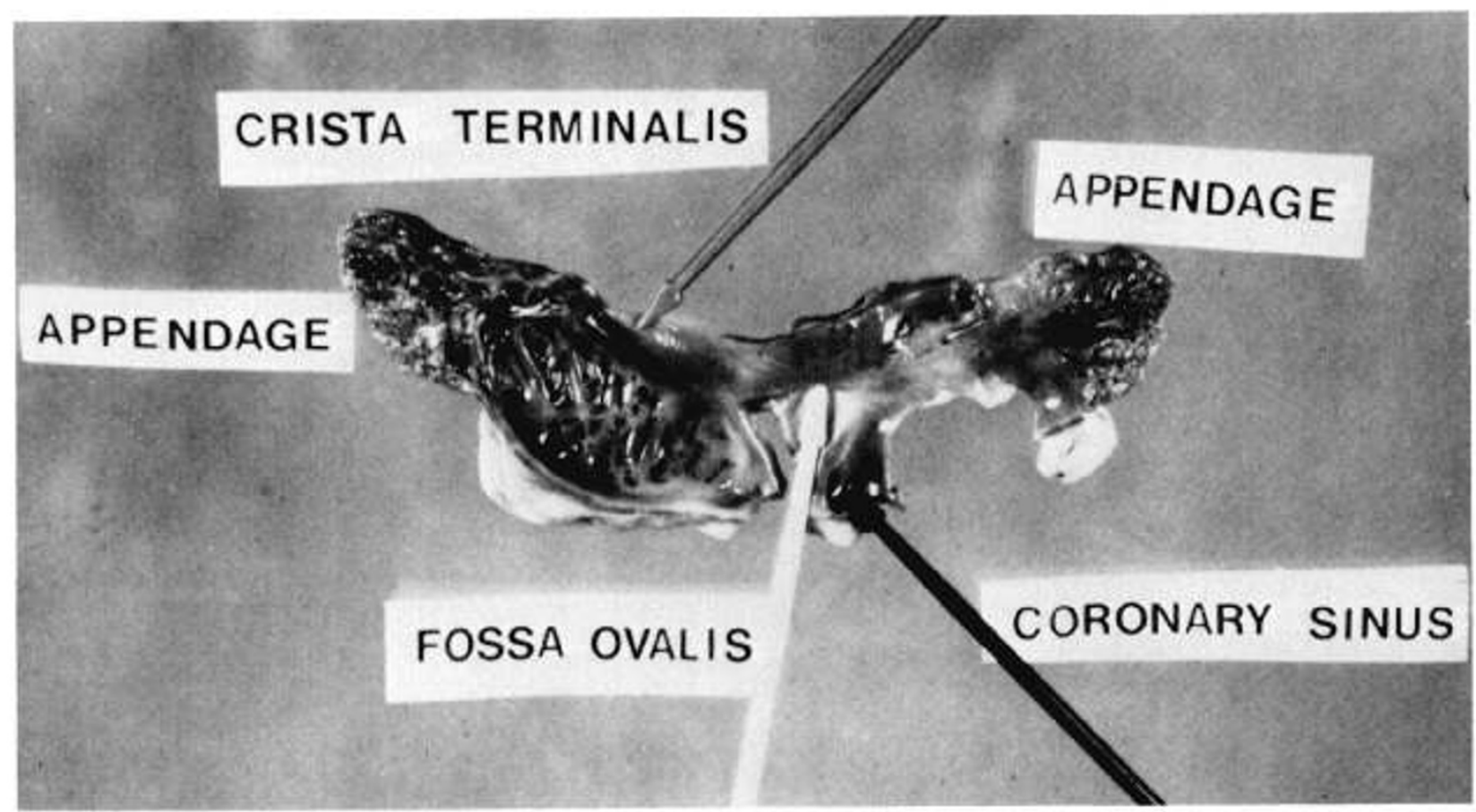

Fig. 1. Photo of the interior of the canine right atrium before sectioning. Arrows point to various anatomical landmarks including the crista terminalis, coronary sinus, and fossa ovalis. Such landmarks were identified routinely in the right atria of humans and dogs and used as a guide to insure consistent sectioning of the atria.

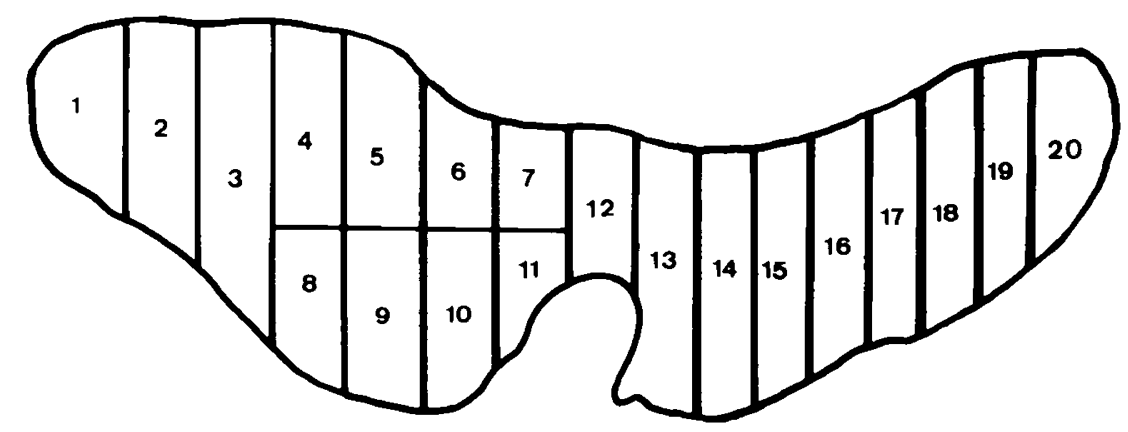

B

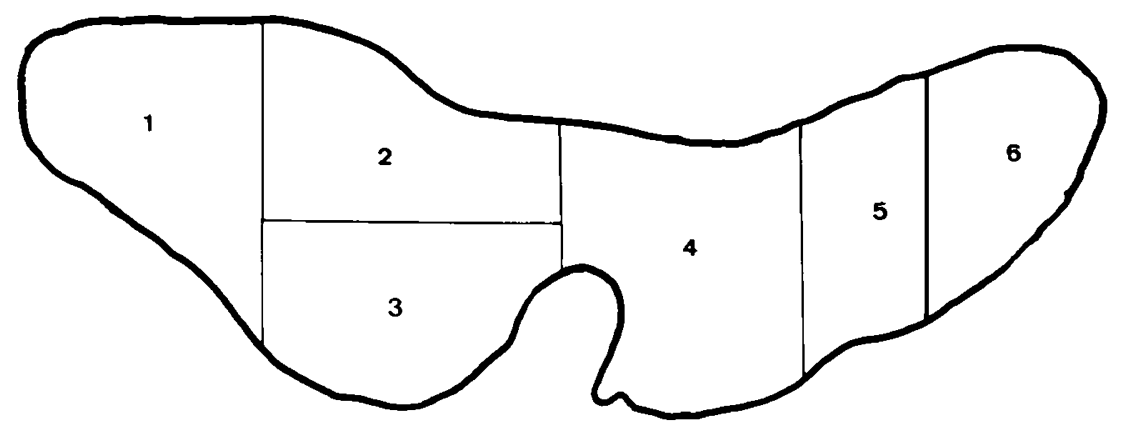

Fig. 2. A Schematic drawing of canine right atrium as shown in Figure 1, indicating how each atrium was cut into 20 sections relative to the various anatomic landmarks described in Methods. $B$ Schematic drawing of the right atrium, as in $A$, indicating the grouping of 20 sections into six areas: Area $1=$ sections $1-3$ inclusive, area $2=$ sections $4-7$, area $3=$ sections $8-11$, ar a $4=$ sections $12-15$, area $5=$ sections $16-17$, area $6=$ sections $18-20$. 
six areas based on the data for sections obtained for dogs (Fig. 3); area 1 (sections $1-3$ inclusive), area 2 (sections 4-7), area 3 (sections $8-11$ ), area 4 (sections 12-15), area 5 (sections 16-17), and area 6 (sections 18-20) as shown in Figure 2B. Area means of right atrial $[\mathrm{AChE}]$ were derived by averaging the appropriate corresponding section values for human infants, human adults, and dogs, respectively. Statistical analysis was accomplished by performing an analysis of variance using the right atrial [AChE] area values for each individual atrium for the three groups studied. F-values having associated $P$-values $\leq 0.01$ were considered statistically significant; multiple comparisons among area means within a group were made using Duncan's multiple range test at the $5 \%$ protection level. Two-tailed $t$ tests were performed when necessary using the appropriate error term extracted from the analysis of variance; for these, $P$ levels $\leq 0.05$ were considered statistically significant.

\section{RESULTS}

The [AChE] distribution found within the canine right atrium is shown in Figure 3 mapped by sections, and in Figure 4 mapped by areas. The [AChE] distribution mapped by areas for human infants and adults are shown in Figure $5 \mathrm{~A}$ and $5 \mathrm{~B}$, respectively. The results of the analysis of variance for the data are summarized in Table 1.

The analysis of variance indicates highly significant differences in mean right atrial $[\mathrm{AChE}]$ among the three groups $(\mathrm{G})$ studied $(F=11.657, P \leq 0.005)$. As determined by $t$ tests $(P \leq 0.05)$ and expressed as $\Delta \mathrm{A} / \mathrm{min} \times 10^{3}$ these differences are: human infants $(60.5)>\operatorname{dogs}(51.4)>$ human adults $(40.5)$. In general, for all three groups combined, there is a nonuniform distribution of [AChE] across the various areas of the right atrium. Statistically, this is highly significant $(\mathrm{F}=5.162, P<0.005)$ as indicated in the analysis for variation among areas (A), Table 1 .

A perusal of Figures 4 and 5 indicates two distinct distribution patterns for right atrial [AChE]. In the dog (Fig. 4), the appendicular areas contain the lowest [AChE] (area 1: 34.3 $\Delta \mathrm{A} / \mathrm{min} \times 10^{3}$; area 6: $33.8 \Delta \mathrm{A} / \mathrm{min} \times 10^{3}$, whereas areas 2 and 4 contain the highest [AChE] (area 2: 62.9 $\Delta \mathrm{A} / \mathrm{min} \times 10^{3}$; area 4: 78.1 $\mathrm{AA} / \mathrm{min}$ $\left.\times 10^{3}\right)$. Multiple comparisons among area means for dogs indicated that only the paired area means $3 v s$. 5 and 1 vs. 6 were not significantly different statistically (5\% protection level of Duncan's multiple range test).
Figure 5 shows the distribution of right atrial [AChE] for human infants (A) and adults (B). The results are shown in terms of area only, by grouping sections as described previously. The [AChE] distribution pattern for human right atria appears to be markedly different from that for dogs. Statistically, this is evident by partition of the distribution term $(A \times G)$ of the analysis of variance (Table 1) into "within species" and "between species" terms. The between species term indicates that the human right atrial distribution of $[\mathrm{AChE}]$ is significantly different from that for the dog $(F=33.402, P \leq 0.005)$; the within species term indicates that there is no statistically significant difference between the distributions of right atrial [AChE] for human infants and adults. Specifically, the appendicular areas contain the highest [AChE]; the latter decreases steadily across the atrium and exhibits its lowest value in area 4 , which represents the interatrial septum and other closely associated landmarks such as the coronary sinus. Multiple comparisons were made to determine statistically significant differences among area means for right atrial [AChE] in human infants and adults (Duncan's multiple range test, 5\% protection level). For human infants, these differences were as follows: area $1>$ areas $2,3,4,5$, and 6 ; area $2>$ areas 4 and 5; area $6>$ areas 4 and 5 . For human adults, the comparisons indicated the following significant differences: area $1>3,4$, and 5 ; area $6>$ area 4 . As noted previously, over the entire right atrium, mean [AChE] for infants is greater than that for adults. This difference is highly significant statistically $(P<0.005)$ as indicated by partitioning the groups $(G)$ term of the analysis of variance into within species and between species terms. However, further analysis utilizing the $t$ test indicates that the [AChE] for each area of human infant right atrium is significantly greater than that for the corresponding area of the adult atrium $(P \leq$ 0.01 ).

\section{DISCUSSION}

The development, ontogenesis, and distribution of both acetylcholinesterase $(\mathrm{AChE})$ and butylcholinesterase $(\mathrm{BuChE})$ in the heart have been studied in many species $(3,5,8,12,13)$ by qualitative histochemical methods. A recent report from this laboratory (11) sought to determine the distribution of [AChE] in various parts of human and dog hearts by quantitative chemical determination of $[\mathrm{AChE}]$ with special reference to species and

\section{ACETYLCHOLINESTERASE CONTENT OF CANINE RIGHT ATRIUM}

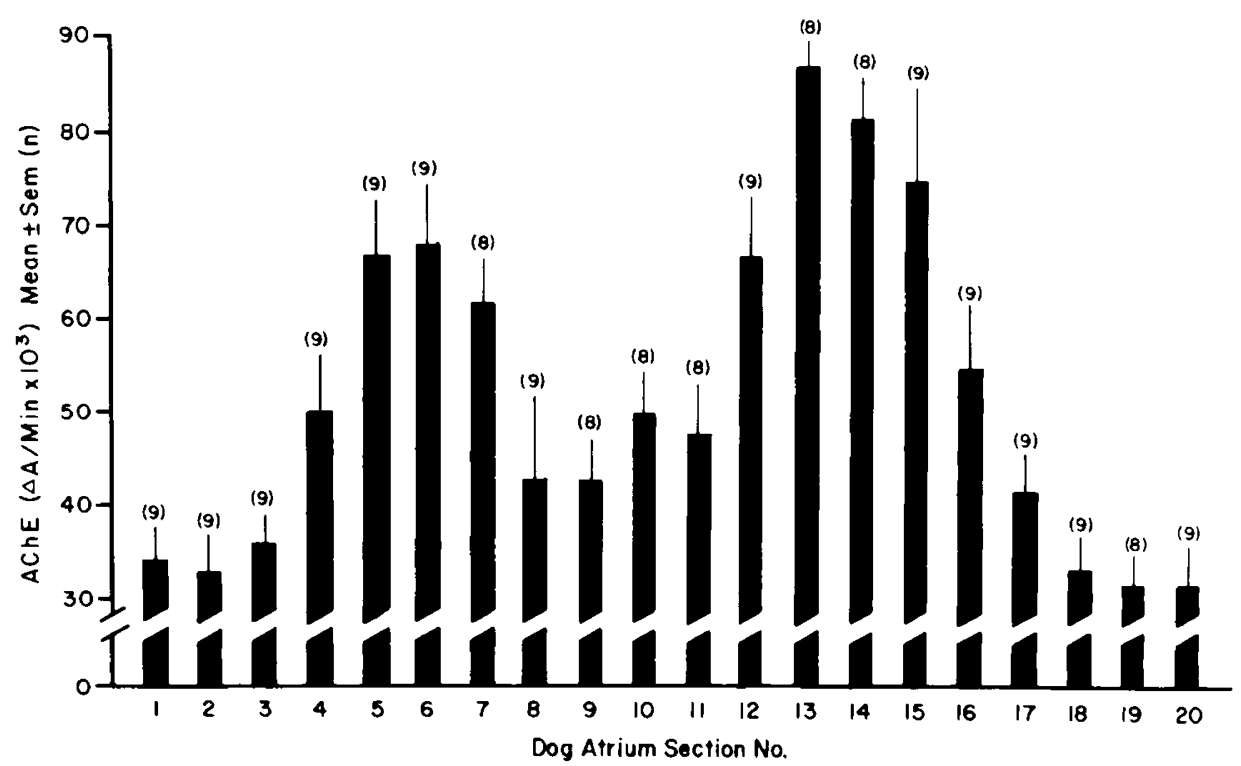

Fig. 3. $[\mathrm{AChE}]$ distribution in the right atrium of nine dogs mapped by section (see Figs $I$ and $2 \mathrm{~A}$ for anatomical correlations; number in parentheses indicates number of tissue samples per mean). 


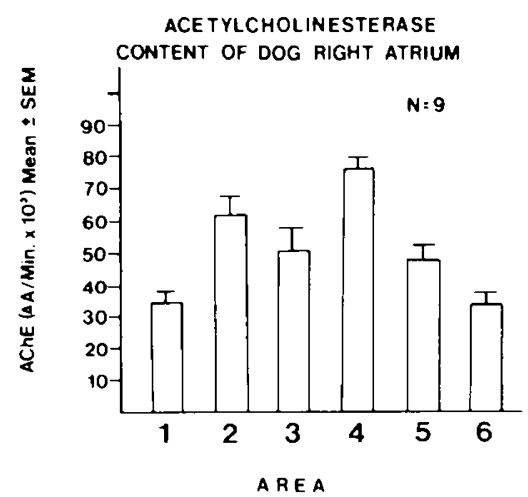

Fig. 4. $[\mathrm{AChE}]$ distribution in the right atrium of nine dogs mapped by area. This distribution was derived from the data of Figure 3 by grouping 2-4 adjacent sections into six areas and obtaining the average [AChE] for each respective area as described in Methods.

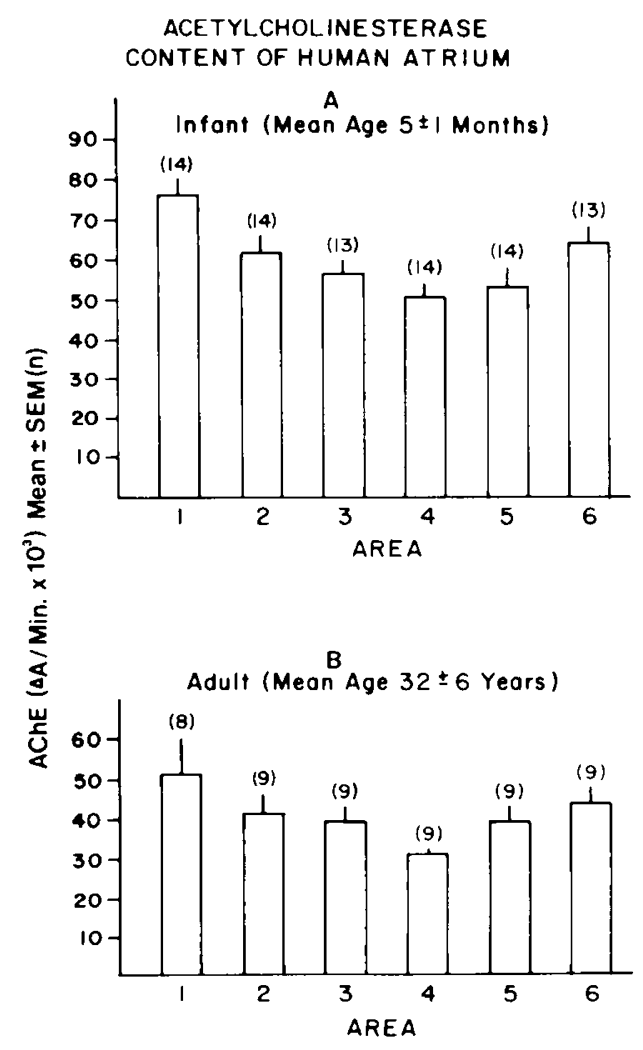

Fig. 5. [AChE] distribution in the right atrium of 14 human infants $A$ and 9 human adults $B$ mapped by area. These distributions were obtained by grouping the $[\mathrm{AChE}]$ values of $2-4$ sections into six areas and obtaining the average [AChE] for each respective area as described in Methods. Numbers in parentheses indicate the number of atria contributing all the necessary tissue samples for a given area.

age-related changes from the neonatal to the adult periods. The main objective of the present study is to extend the previous work and to examine in greater detail the regional distribution of [AChE] within the right atria of human infants and adults, and dogs.

In the previous study (11), it was demonstrated that in the neonatal period, [AChE] in the atrial appendage was over four times greater in human infants than in puppies. Postnatal changes were found to minimize this difference as a result of declining enzyme concentration in the human right atrium and increasing enzyme concentration in the dog right atrium (11). The present findings reaffirm this relationship in that with respect to age in the human, the appendage [AChE] (areas 1 and 6) is higher for infants $\left(70 \Delta \mathrm{A} \times 10^{3} / \mathrm{min}\right.$, average of means for areas 1 and 6$)$ than for adults $\left(47 \Delta \mathrm{A} \times 10^{3} / \mathrm{min}\right)$. Indeed, all areas of infant atria demonstrated higher [AChE] than corresponding adult areas. In addition, atrial appendage $[\mathrm{AChE}]$ for $\operatorname{dogs}\left(34 \Delta \mathrm{A} \times 10^{3} / \mathrm{min}\right.$; average of areas 1 and 6) closely approximates that for adult humans (47 $\left.\Delta \mathrm{A} \times 10^{3} / \mathrm{min}, P>0.05\right)$ as noted previously (11).

The distribution of [AChE] across the right atria of both humans and dogs was found to be nonuniform. However, the [AChE] distribution pattern was markedly different between the two species. For humans, the appendicular areas (areas 1 and 6) contained the greatest [AChE]. [AChE] declined gradually across the atrium, having its lowest value in the area of the interatrial septum (area 4). In the dog, $[\mathrm{AChE}]$ was lowest in areas 1 and 6 , while areas 2 and 4 contained the highest [AChE].

The species and age-related differences in the [AChE] distribution are real and do not occur as a result of the differing time intervals between death and the analysis of tissue samples. Evidence for this is as follows: First, canine atrial tissue samples were analyzed both at death and after storage in $0.9 \%$ saline for up to $24 \mathrm{hr}$ at room temperature. No significant difference in [AChE] was noted between fresh and stored canine atrial tissues. Second, a statistical correlation analysis was performed to test the relationship of [AChE] of all areas of human atria to the time to postmortem analysis of the tissue samples. A correlation coefficient $(r)$ for adult human was 0.132 (computed $P=0.376$ ) and that for infants was 0.093 (computed $P=0.406$ ). Thus, tissue [AChE] measurements in humans do not appear to be affected by the unavoidable delay between death and analysis. These age- and species-related differences were not unexpected based on our previous work (11). In addition, the species-related differences are further supported by the observation that similar [AChE] distribution patterns are obtained for both populations of humans (adults and infants), even though infant atria contained significantly greater $[\mathrm{AChE}]$ than adult atria.

The $[\mathrm{AChE}]$ distribution in the dog right atrium is very interesting considering the physiologic phenomena that have been observed for the canine atrium. Alessi et al. (2) have shown the effects of direct and reflex vagal stimulation on the refractory period (RP) for three points on the dog right atrium. They found the effects of vagal stimulation on RP were not uniformly distributed over the atrium. During stimulation of the nerves, there were marked effects on RP at some points, while no effects on RP were apparent at other points when the vagus nerves were stimulated singly or together. Unfortunately, this paper presents only selected examples of the experiments and does not specifically define the points of measurements accurately enough in the anatomical sense, thus correlations of the present chemical study with the electrophysiologic study are extremely difficult. However, it is

Table 1. Analysis of variance for right atrial [AChE] by areahuman infants, human adults, and dogs

\begin{tabular}{lrrrrc}
\hline \multicolumn{1}{c}{ Source } & df & \multicolumn{1}{c}{ SS } & \multicolumn{1}{c}{ MS } & \multicolumn{1}{c}{ F } & \multicolumn{1}{c}{$P$} \\
\hline Groups $\left(\mathrm{G}_{\mathrm{B}}\right)$ & 2 & $12,785.8407$ & $6,392.9203$ & 11.657 & $<0.005$ \\
$\quad$ Within species & 1 & $12,710.8233$ & $12,710.8233$ & 23.176 & $<0.005$ \\
$\quad \begin{array}{l}\text { Between spe- } \\
\quad \text { cies }\end{array}$ & 1 & 75.0174 & 75.0174 & 0.137 & NS \\
$\begin{array}{l}\text { Blocks (B) } \\
\text { Areas (A) }\end{array}$ & 29 & $15,904.7530$ & 548.4398 & 5.087 & $<0.005$ \\
$\begin{array}{l}\text { Distribution } \\
\quad \text { A } \times \text { G) }\end{array}$ & 10 & $18,782.7234$ & 556.5447 & 5.162 & $<0.005$ \\
$\quad$ Within species & 5 & 299.5393 & 53.9079 & 0.556 & NS \\
$\quad$ Between spe- & 5 & $18,006.4215$ & $3,601.2843$ & 33.402 & $<0.005$ \\
$\quad$ cies & & & & & \\
Error & 145 & $15,633.5423$ & 107.8175 & & \\
Total & 191 & $65,412.8202$ & & & \\
\hline
\end{tabular}


possible that the nonuniform vagal effects may have a parallel to, and to some extent are reflected by the nonuniform distributions of [AChE] demonstrated for the canine right atrium in the present study.

It has been shown that good correlations exist between the concentrations of acetylcholine, acetylcholinesterase, and choline acetylase in various regions of the peripheral and central nervous systems (9). Thus, while the presence of AChE in the heart does not necessarily implicate the concomitant association of function of acetylcholine, one can conjecture that the [AChE] distribution within the atrium corresponds to the cholingergic nerve distribution there. Therefore, the sinoatrial node and the atrioventricular node would be expected to have dense cholinergic innervation, and hence would be expected to demonstrate high [AChE]. The results of the present study bear out such a relationship in reference to the dog, as the areas with the highest [AChE] encompass the sinoatrial and atrioventricular nodes (areas 2 and 4), respectively.

In this regard, a very interesting relationship has been realized as a result of the present study and another by Coglianese $e t$ al. (6) at this institution. They determined the relative densities of sympathetic nerve terminals in canine right atria by following ${ }^{3} \mathrm{H}$ norepinephrine uptake. Atria were sectioned and numbered exactly as in the present study, and it was determined that the neurally intact atria displayed a consistent gradient of ${ }^{3} \mathrm{H}$-norepinephrine uptake, which was abolished by chronic cardiac denervation. Uptake was highest at the extreme tip of the two appendicular areas, declining gradually towards the interatrial septum, where uptake was lowest. These latter results, in concert with the [AChE] distribution observations reported here for dogs, can be construed as indicating a reciprocal sympathetic-parasympathetic innervation of the dog right atrium. Thus, where sympathetic innervation is high, parasympathetic innervation is low.

At the present time, no definitive statements can be made to explain the difference in the $[\mathrm{AChE}]$ distribution patterns demonstrated between human and dog right atrium. One possible explanation is that the cholinergic innernation to the right atrium is different between the two species. These differences may indicate that the distribution of vagal effects on the human atrium vary considerable from those for dogs (2). Such differences may have far reaching implications in the clinical management of atrial arrhythmias in the human. As cardiac function is dependent on both the sympathetic and parasympathetic divisions of the autonomic nervous system, further studies, similar to the present studies, are needed to substantiate the regional distribution of the sympathetic nerve terminals within the human right atrium, in order to provide the basis for a better understanding of the functional innervation of the human heart.

The age-related differences demonstrated between human in- fants and adults most likely reflect maturational changes, the significance of which have been discussed previously (11).

\section{REFERENCES AND NOTES}

1. Aldridge, W. N.: The differentiation of true and pseudocholinesterase by organophosphorous compounds. Biochem. J. 53: 62 (1953).

2. Alessi, R., Nusynowitz. M., Abildskov, J. A., and Moe, G. K.: Non-uniform distribution of vagal effects on the atrial refractory period. Am. J. Physiol., 194 (2): 406 (1958)

3. Antopol, W., Gilauback. S., and Gilick, D.: Choline esterase activity in various portions of the rabbit heart. Proc. Soc. Exp. Biol. Med., 42: 280 (1939)

4. Austin, L., and Berry, W. K.: Two selective inhibitors of cholinesterase. Biochem. J., 54: 695 (1953).

5. Bojseu-Moller, F., and Tranum-Jensen. J.: Wholemount demonstration of cholinesterase containing nerves in the right atrial wall, nodal tissue, and atrioventricular bundle of the pig heart. J. Anat.. 108: 375 (1971).

6. Coglianese, C., Randall, W. C., and Filkins, J. P.: The relative density of sympathetic nerve terminals in the canine right atrium. Proc. Soc. Exptl. Biol Med.. 154: 127 (1977).

7. Ellman, G. E., Courtney, B. D., Andres, V., and Featherstone, R. M.: A new rapid colorimetric determination of acetylcholinesterase activity. Biochem. Parmacol., 7: 88 (1961).

8. Holmes, R. L.: Cholinesterase activity in the atrial wall of the dog and cat heart. J. Physiol., 137: 421 (1957).

9. Jacobowitz, D., and Koelle, G. G.: Histochemical correlations of acetylcholinesterase and catecholamines in postganglionic autonomic nerves of the cat, rabbit and guinea pig. J. Pharmacol. Exptl. Ther., 148: 225 (1965).

10. Karczmar, A. G., Srinivasan. R.. Bernsohn, J.: Cholinergic function in the developing fetus. In: L. Borius: Fetal Pharmacology pp. 127-177 (RaveriPress, New York. 1973.

11. Sinha, S. N., Keresztes-Nagy, S., and Frankfater. A.: Studies on the distribution of cholinesterases activity in the human and dog heart. Pediatr. Res.. 10: 754 (1976).

12. Sippel. T. O.: Properties and development of cholinesterase in the hearts of certain vertebrates. J. Exp. Zool., 128: 165 (1955).

13. Truex, R. C., Scott. J. C., Long D. M., and Smyth, M. Q.: Effect of vagus nerves on heart rate of young dogs. An anatomic-physiologic study. Anat. Res., 123: 201 (1955)

14. Informed consent of nearest relative was obtained before use of any human tissues.

15. The authors thank Mr. Merl Kardatzke, who assisted in the statistical analysis The authors wish to express their sincere thanks to Dr. Walter Randall for his valuable suggestions and criticisms of the work and in the preparation of this manuscript. Also, thanks to Monica Powers for her invaluable technical assistance and to Beatrice Lorr, Diane Konrad, and Luanne Fassero for their expert secretarial assistance in preparing this manuscript.

16. The rate expressed this way may be converted to International Units (IU = $\mu$ mole of substrate hydrolyzed $/ \mathrm{min} / \mathrm{ml}$ ) according to the following equation:

$$
\mathrm{IU} / \mathrm{g} \text { tissue }=\frac{\Delta \mathrm{A} / \mathrm{min}}{13.6} \times \frac{(\text { Total assay volume })}{(\text { Sample volume })} \times 10
$$

17. This research was supported by the National Institutes of Health Grant \#1R01HD08465-01 Al and the Chicago Heart Association.

18. Requests for reprints should be addressed to: S. N. Sinha. M.D., Department of Pediatrics, Peoria School of Medicine. c/o St. Francis Hospital, Peoria, Illinois 61637 USA.

19. Received for publication July 20, 1977.

20. Accepted for publication October 12, 1978 